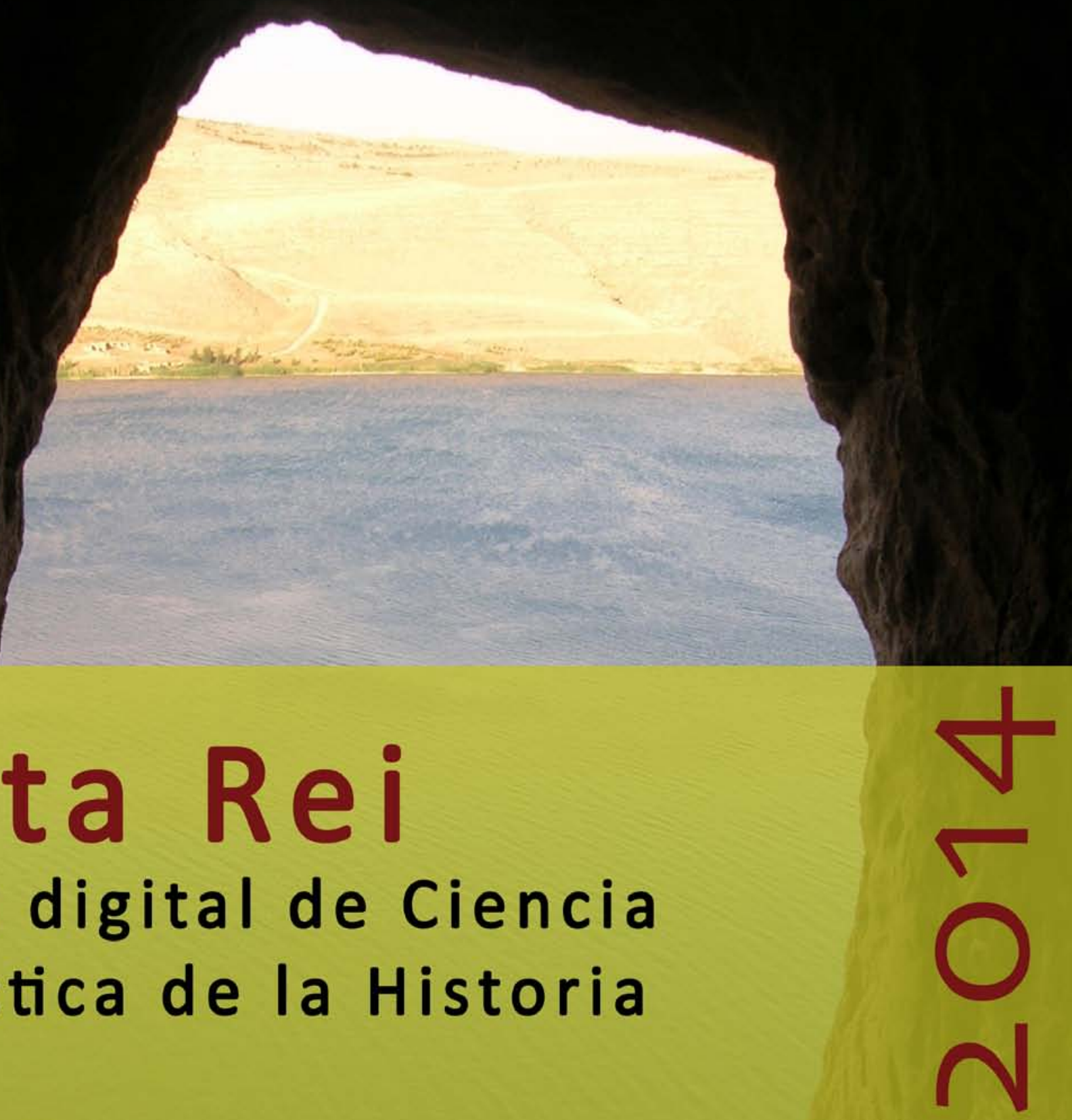





\section{Panta

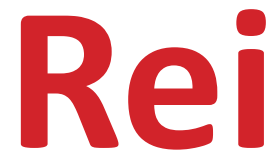 \\ Revista Digital de Ciencia \\ y Didáctica de la Historia}

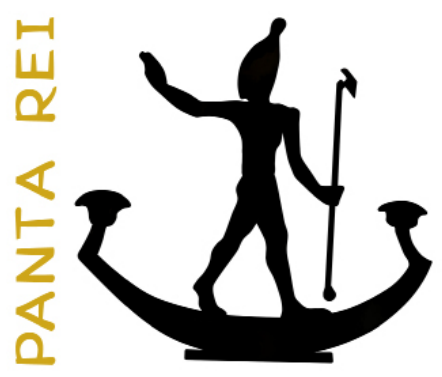

2014

Revista anual

Fecha de inicio: 1995

Revista Panta Rei. pantarei@um.es

\section{Edita:}

Centro de Estudios del Próximo Oriente y la

Antigüedad Tardía - CEPOAT

Edificio Universitario Saavedra Fajardo.

Universidad de Murcia

C/ Actor Isidoro Máiquez, 9

30007 - MURCIA - ESPAÑA

Teléfono: (+34) 868883890

cepoat@um.es

Web: www.um.es/cepoat/pantarei

Edición 2014

ISSNe: 2386-8864

ISSN: $1136-2464$

Depósito legal: MU-966-1995
cepoAt

UNIVERSIDAD DE MURCIA centro de estudios del próximo oriente y la antigüedad tardia

En Portada: Río Éufrates desde un eremitorio cristiano primitivo (Fotografía de Alejandro Egea).

Responsables de los textos:

Sus autores.

Responsable de la presente edición: Consejo Editorial Panta Rei. 


\section{CONSEJO DE REDACCIÓN}

\section{Coordinador editorial}

Egea Vivancos, Alejandro

[Didáctica de las Ciencias Sociales, UMU]

\section{Editores}

Botí Hernández, Juan Jesús

[CEPOAT, UMU]

Sáez Giménez, David Omar [CEPOAT, UMU]

\section{Secretaria}

Arias Ferrer, Laura

[Didáctica de las Ciencias Sociales, UMU]

\section{Responsable informático}

Martínez García, José Javier

[CEPOAT, UMU]

\section{Traducción y corrección lingüística}

Martínez Martínez, Cristina

[Sociedad Española de Lenguas Modernas]

\section{CONSEJO ASESOR}

Albero Muñoz, M. ${ }^{a}$ del Mar [H. ${ }^{a}$ del Arte, UMU]

Cobacho López, Ángel

[Derecho, UMU]

Egea Bruno, Pedro M. ${ }^{a}$

[Historia Contemporánea, UMU]

García Atienzar, Gabriel

[Prehistoria, UA]

González Monfort, Neus

[Didáctica de las Ciencias Sociales, UAB]

Haber Uriarte, María

[Prehistoria, UMU]

Irigoyen López, Antonio

[Historia Moderna, UMU]

Mahony, Simon

[Digital Humanities, UCL, Reino Unido]

Marsilla de Pascual, Francisco Reyes

[Técnicas historiográficas, UMU]

Miralles Maldonado, José Carlos

[Filología Clásica, UMU]

Molina Gómez, José Antonio

[Historia Antigua, UMU]

Noguera Celdrán, José Miguel

[Arqueología, UMU]

Pérez Molina, Miguel Emilio

[Filología Clásica, UMU]

Prados Martínez, Fernando

[Arqueología, UA]

Sánchez Ibáñez, Raquel

[Didáctica de las Ciencias Sociales, UMU]

Sancho Gómez, Miguel Pablo

[Educación, UCAM]

Vilar García, María José

[Historia Contemporánea, UMU] 
Presentación

Todo fluye. Renovarse y crecer

Alejandro Egea Vivancos, Laura Arias Ferrer, Juan Jesús Botí Hernández y David Omar Sáez Giménez

Artículos

La sociedad hiperbórea: ¿utopía o mito? Reflexiones acerca de la naturaleza y significado del relato hiperbóreo.

José Ángel Castillo Lozano.

Aproximación a la figura de una matrona romana culta y poderosa. El caso de Julia Domna.

Consuelo Isabel Caravaca Guerrero.

¡Arrasar la Vendée! Guerra Civil y Columnas Infernales en pleno corazón de la Revolución Francesa.

Benjamín Cutillas Victoria.....

Arte y expresión en el pensamiento de E. H. Gombrich.

Patricia Castiñeyra Fernández.

Odiseo a través de la parodia. Desmitificación e ironía de una Ítaca nostálgica en Prometeo de Pérez de Ayala y ¿Por qué corres, Ulises? de Gala.

Carmen María López López.

Análisis de la actividad didáctica del Museo de Arte Ibérico El Cigarralejo (Mula, Murcia).

Julio García Toral......

La Transición española en $4 .^{\circ}$ ESO. Un estudio de caso de los significados.

Borja Santiago Arnoso.

Entrevista

Entrevista al profesor D. Michael Walker.

Consuelo Isabel Caravaca Guerrero.

Reseñas

II Congreso de la Asociación Internacional de Investigación para la Educación de la Historia y las Ciencias Sociales.

Elvira Barriga y Rodrigo Salazar.

I Congreso de Jóvenes Investigadores del Mundo Antiguo de la Universidad de Murcia.

Pedro David Conesa Navarro

II Congreso Internacional de Educación Patrimonial.

Tània Martínez Gil.....

Ruiz-Gálvez Pliego, M. (2013). Con el fenicio en los talones. Los inicios de la Edad del Hierro en la cuenca del Mediterráneo. Barcelona: Editorial Bellaterra. 377 págs.

Celso Sánchez Mondéjar..

Normas de publicación/Publishing rules 



\title{
Entrevista al profesor D. Michael Walker
}

\author{
Consuelo Isabel Caravaca Guerrero \\ Universidad de Murcia
}

Para citar esta entrevista: Caravaca Guerrero, C. I. (2014, 15 de enero). Entrevista al profesor D. Michael Walker. Panta Rei. Revista Digital de Ciencia y Didáctica de la Historia, 135-146. ISSNe: $2386-8864$

DOI: http://dx.doi.org/10.6018/pantarei/2014/9

Entrevistar a un investigador de la talla de D. Michael Walker supone hablar de un investigador reconocido a nivel nacional e internacional, uno de los mayores expertos en evolución humana de nuestros días. Nació en Colchester (Inglaterra) en 1941 y tiene doble nacionalidad: británica y australiana. Actualmente es profesor emérito de la Universidad de Murcia en el Departamento de Zoología y Antropología Física de la Facultad de Biología. Pero su currículum no acaba aquí, Michael realizó tres carreras, Fisiología Animal, Medicina, y Arqueología Prehistórica, en la Universidad de Oxford. Es Doctor por esta universidad, gracias a su tesis leída sobre Paleoantropología y Prehistoria de las cuencas de los ríos Segura y Vinalopó. Nuestro entrevistado, también fue elegido Académico (Fellow -F.S.A.-) en 1988 de la Society of Antiquaries of Londono SAL. Fundada en 1707, la SAL es la equivalente británica de la española Real Academia de la Historia. Es Fellow también del Real Instituto Antropológico de la Gran Bretaña (Royal Anthropological Institute of Great Britain) y socio emérito de la Asociación Americana de Antropólogos Físicos (American Association of Physical Anthropologists). Suele presentar sus investigaciones ante reuniones científicas de carácter internacional y publicar artículos científicos en prestigiosas revistas internacionales principalmente, también emite publicaciones a nivel nacional o meramente regional. La evolución de su carrera científica de profesor universitario e investigador se desarrollaba a través del ejercicio de la labor académica en las universidades de Oxford, Edimburgo, Sydney, y la Autónoma de Madrid. Desde hace 25 años dirige las excavaciones de la Cueva Negra del Estrecho del Quípar (Caravaca) y de Sima de las Palomas del Cabezo Gordo (Torre Pacheco). Actualmente es el Presidente de MUPANTQUAT (Asociación Murciana para el Estudio de la Paleoantropología y el Cuaternario) y realiza trabajos de investigación en Grupo de Investigación E005-11 de la Universidad de Murcia Paleoecología, Paleoantropología y Tecnología del Cuaternario establecido desde el 1 de enero del 2014 al unirse los anteriores grupos Tecnología, Antropología y Ecología del Cuaternario y Palinología y Estudios Paleoambientales. Se casó en 1968 con la murciana M. ${ }^{\text {a Teresa Pina }}$ Velasco, tristemente fallecida en 1998, y cuenta entre sus familiares más cercanos con tres hijos y cuatro nietos. Como vemos una vida en constante movimiento e investigación, con un currículum nada desdeñable. 
C.I.C.G.: Ciñéndonos a la época más cercana y con respecto a las excavaciones, ¿Cuáles fueron los motivos que le llevaron a interesarse por estos enclaves arqueológicos? ¿Destacaba en ellos alguna característica especial? ¿Los conocía previamente o fue necesario prospectar la zona? Hable según cada caso.

M. Walker: Con respecto a Cueva Negra del Estrecho del río Quípar, Caravaca de la Cruz, la cual está fechada entre 990000 y 780000 años, en 1986 el arqueólogo Miguel San Nicolás del Toro me enseñó la Cueva Negra donde en 1981 con Miguel Martínez Andreu y Ricardo Montes Bernabé (ambos ahora doctores en Arqueología) realizó una breve prospección. En 1986 tenía permiso de viaje en comisión de servicio por la Universidad de Sydney donde fui profesor. Vine a España en condición de Catedrático Becado por la Comisión Interministerial de Ciencia y Tecnológica (CICyT), adscrito para doce meses a la Facultad de Ciencias de la Universidad Autónoma de Madrid donde impartía clases de especialización de carrera. También colaboraba con la Facultad de Biología de la Universidad de Murcia con la impartición de tres ciclos de clases sobre Evolución Humana: para principiantes, de especialización de carrera, y de postgrado. En 1988 me incorporé en la plantilla docente permanente de nuestra Facultad de Biología, a través del Programa PROPIO (Programa de Oferta de Plazas con la Investigación como Objetivo) del Ministerio de Educación y Cultura ante el cual me había propuesto la Universidad de Murcia para ser profesor permanente de nacionalidad extranjero a nivel de Catedrático de Universidad, de acuerdo con la previsión de la Disposición Adicional Octava de la Ley Orgánica de Reforma Universitaria del 1983, puesto que todavía en 1988 los extranjeros fuimos vetados para opositar para los Cuerpos Docentes Universitarios por otra Ley Orgánica que regulaba los Cuerpos de Funcionarios del Estado Civil Español; sin embargo, la Disposición 8. ${ }^{a}$ indicaba que el nombramiento tuviera el visto bueno de la Conferencia de Rectores de Universidades Españolas o CRUE. Conviene rebobinar la historia para aclarar que las circunstancias de mi acceso a la plantilla docente permanente, habilitado a nivel de Catedrático de Universidad. El último catedrático extranjero en la Universidad española (la de Madrid) fue el prehistoriador austríaco Hugo von Obermaier, autor del Hombre Fósil en España, que cuando estalló la Guerra Civil se retiró a la católica universidad suiza de Freiburgo donde murió en 1946. Luego se creó una fundación para conmemorar su obra y la Asociación Internacional Obermaier para la Investigación del Paleolítico y de la Edad del Hielo (Hugo Obermaier-GesellschaftfürErforschung des Eiszeitaltersund der Steinzeit) de la que soy socio y en nuestra 54. ${ }^{a}$ reunión anual del 2012 presenté "El Hombre Fósil en el Sudeste de España". Después de aterrizar en Murcia me enteré que nuestra amiga la Dra. Ana M. ${ }^{a}$ Muñoz Amilibia había apoyado con energía la elevación de la propuesta en mi favor ante las autoridades de nuestra Universidad. No obstante, pese a mi anterior dedicación científica al estudio de la Prehistoria y Paleoantropología del Holoceno murciano quería evitar cualquier sospecha de pretensión de hacer competencia desleal, por lo que estimé que mi atención a la Evolución Humana podría desarrollarse oportunamente en el Pleistoceno, período casi abandonado en aquellos momentos por el profesorado de nuestra Universidad. Consideré la Cueva Negra un yacimiento excelente para la investigación de los sedimentos del Pleistoceno (expuestos por el trabajo de 1981) y la formación científica correspondiente de alumnos y jóvenes científicos de nuestra Universidad. En 1990, con el visto bueno del propietario y el permiso oficial de la Dirección General de Cultura, inicié la excavación sistemática de la cueva. Desde entonces han sido realizadas 24 campañas anuales. Los primeros hallazgos habían suscitado la posibilidad de un conjunto paleolítico y paleontológico de apenas 100.000 años de antigüedad, o sea del comienzo del Pleistoceno Reciente (antes llamado Pleistoceno Superior). Sin embargo, la evolución de la investigación ha demostrado que todo el conjunto tiene casi un millón de años -anterior a 780000 pero posterior a 990 000- al final del Pleistoceno Antiguo (antes llamado Pleistoceno Inferior) pero antes del inicio del Pleistoceno Medio hace 780000 años. Hemos recuperado dientes del Homo heidelbergensis aff. neanderthalensis, que fue un ancestro muy lejano de los neandertales. Hemos excavado el hacha de mano achelense más antigua de Europa y también hemos descubierto una evidencia contundente de la fogata más antigua del Paleolítico europeo. 
Con respecto a Sima de las Palomas del Cabezo Gordo, Torre Pacheco, su antigüedad se sitúa sobre los 50000 años. La existencia de un afloramiento de brecha expuesta en la ladera del Cabezo Gordo, con la exposición de huesos fósiles del Cuaternario, me fue comunicada por el ilustre geólogo y paleontólogo D. Emiliano Aguirre Enríquez, catedrático de la Universidad Complutense. En 1989, inspeccionó el afloramiento D. Miguel San Nicolás del Toro, arqueólogo técnico del entonces denominado Centro Regional de Arqueología de la Comunidad Autónoma de la Región de Murcia (actualmente el Servicio de Patrimonio Histórico de la Dirección General de Bellas Artes y Bienes Culturales de la Consejería de Cultura) acompañado por integrantes de la Coordinadora Ecologista para la Defensa del Cabezo Gordo. Ellos le informaron sobre la existencia de otras estaciones de brecha fosilífera en la solana del Cabezo, aunque no pudo visitarlas aquel día por falta de tiempo. Unos meses después, en 1990, visité el afloramiento en compañía de Miguel San Nicolás del Toro, el doctor Roque Ortiz Silla, geólogo y catedrático de la Universidad de Murcia, el doctor Antonio Ruiz Bustos (a sugerencia de D. Emiliano Aguirre) paleontólogo y profesor titular de la Universidad de Granada, y D. Antonio Sánchez Garnés, copropietario de la empresa marmolista "Cabezo Gordo S.A." y copropietario de la finca del Cabezo Gordo. Como consecuencia de la visita, en enero de 1991 presenté un escrito ante el Centro Regional de Arqueología en el que se recomendaba la realización, con carácter de urgencia, de un programa de proyectos de prospección, rescate y estudio paleontológico de las estaciones fosilíferas y brechas del Cabezo Gordo. El escrito fue motivo de una reunión informal, celebrada en Murcia en el propio Centro Regional de Arqueología, en la que participé, además de D. Antonio Sánchez Garnés, el gerente de la empresa "Cabezo Gordo S.A.", D. Miguel Pérez Manzanera, y dos arqueólogos técnicos del Centro, Miguel San Nicolás del Toro y Ángel Iniesta Sanmartín. La reunión acordó que no sólo era oportuno, sino también recomendable y deseable, realizar semejante programa científico en el Cabezo, pero la falta de recursos económicos disponibles en la fecha de la reunión aconsejó aplazar la presentación del proyecto hasta el otoño, período más adecuado porque en ese trimestre se confeccionaría el presupuesto para el ejercicio económico del año siguiente, 1992. Sin embargo, la aparición en la prensa regional de noticias en torno a la presencia de estaciones fosilíferas en el Cabezo Gordo puso de relieve el peligro que corrían a manos de la depredación y del expolio ignorante o, peor incluso, para el lucro interesado privado. En noviembre de 1991, D. Antonio Sánchez Garnés en nombre de la empresa y la propiedad, remitió al Director General de Cultura un escrito firmado que indicaba la continuada voluntad de la propiedad de que yo era autorizado por ellos para llevar a cabo todas las investigaciones científicas necesarias con un equipo dirigido desde la Universidad de Murcia. Mientras tanto, durante el verano de 1991 cuando me hallaba ocupado dirigiendo la segunda campaña de excavación oficial en la Cueva Negra del Estrecho del Río Quípar en Caravaca, algunos jóvenes de la citada Coordinadora Ecologista habían recogido de la brecha en la Sima de las Palomas, sin autorización, algunos huesos fósiles de un leopardo y un fragmento de conglomerado fosilífero en el que se apreciaban dientes parecidos a incisivos humanos de tipo Homo neanderthalensis y los entregaron a mi amigo el científico Josep Gibert Clols, paleontólogo con notable trayectoria científica del Institut Paleontológic "Dr. M. Crusafont" de la Diputación de Barcelona en Sabadell, que se encontraba dirigiendo la excavación paleontológica oficial de la Cueva Victoria, en el Llano del Beal de Cartagena. El Centro Regional de Arqueología en seguida concedió permiso escrito a Josep para efectuar una prospección en el lugar del hallazgo, con eficacia supuesta para abarcar la extracción de los hallazgos anteriores, sin pararse a pensar en la oportunidad de convocar otra reunión equiparable a aquella celebrada meses atrás, esta vez con la incorporación adicional del paleontólogo barcelonés, antes de la toma de una decisión administrativa contundente. Dicha autorización concedida precipitadamente no pudo ser activada, entre otras razones, porque faltaba el permiso escrito de la propiedad, requisito obligado e ineludible para efectuar semejante labor investigadora de campo según las normas vigentes del propio Centro Regional de Arqueología, dado que los propietarios de la finca del Cabezo habían reiterado su voluntad, ya expresa por escrito y en la reunión en el Centro, de que la realización de la investigación de las brechas fosilíferas de la propiedad fuera acometida por mí. Conocía a Josep 
desde 1975 cuando coincidimos en Jaca en la Segunda Reunión Nacional del Grupo Español de Trabajo del Cuaternario celebrada en el Instituto de Estudios Pirenaicos del Consejo Superior de Investigaciones Científicas, y luego nos veíamos a menudo bien en Sabadell o en sus excavaciones en Orce (Granada). Así pues, no fue difícil resolver el asunto del Cabezo Gordo oportunamente; nos comprometimos a dirigir los trabajos de investigación conjuntamente. La consiguiente expedición en 1992 de nuestros permisos oficiales por la Dirección General de Cultura marcó el inicio de 22 campañas anuales hasta la fecha. La colaboración científica fue fructífera, la amistad se profundizó y el avance de la investigación se extendió año tras año. Tristemente, nuestro amigo Josep el ilustre investigador de Sabadell falleció en 2007 cuando apenas habíamos empezado a descubrir los estupendos esqueletos articulados de los tres neandertales (Homo neanderthalensis), uno de ellos lo hemos bautizado como "Paloma" y pareja con su niño, enterrados con útiles musterienses hace 50000 años durante el Pleistoceno Reciente (antes llamado Pleistoceno Superior). No es usual hallar esqueletos individuales en conexión anatómica de tamaña antigüedad. La familia neandertal murciana pronto descansará al pie del Cabezo Gordo en el impresionante regional Museo de Paleontología y Evolución Humana, actualmente en fase de determinación interior para acomodarla.

C.I.C.G.: Sobre Cueva Negra, ¿Nos podría mencionar que tipo de restos arqueológicos han aparecido, si hay restos líticos, huesos humanos, fauna etc?

M.Walker: Han sido identificados media docena de dientes humanos del Homo heidelbergensis aff. Neanderthalensis, el cual es el precursor lejano de hace unos 800000 años en Cueva Negra de los neandertales que sólo aparecen hace 150000 en el registro de la Evolución Humana. Los restos de fauna son muy abundantes, tanto mamíferos mayores y menores, como reptiles, anfibios, peces e incluso ¡66 aves!. El análisis del polen realizado por el Catedrático de Evolución Vegetal Dr. José Carrión pone de relieve la presencia de bosque de galería con árboles hoy en día escasos o ausentes. Tanto el polen como la fauna, especialmente las 7 especies aves acuáticas, nos hablan de un entorno desaparecido de pantanos y lagos cerca de la cueva. La cueva estaba entonces en el centro de un vergel de notable biodiversidad, con alimentos comestibles de todo tipo al alcance de los visitantes o moradores del abrigo. Los restos paleolíticos incluyen un hacha de mano achelense y numerosos utensilios pequeños sobre lascas y fragmentos (de sílex, caliza, cuarcita) con extracción centrípeta repetida, a veces sobre núcleos discoideos pequeños (por la técnica Levallois), y a veces con retoque marginal abrupto (musteroide), por lo que hemos designado el conjunto lítico achelense-levaloisense-musteroide véase M. J. Walker, M. V. López Martínez, J. S. Carrión García, T. Rodríguez Estrella, M. San Nicolás del Toro, J. L. Schwenninger, A. López Jiménez, J. Ortega Rodrigáñez, M. Haber Uriarte, J. L. Polo Camacho, J. García Torres, M. Campillo Boj, A. Avilés Fernández, W. Zack (2013).

\section{C.I.C.G.: ¿Los utensilios líticos están realizados con material autóctono o es foráneo?}

M.Walker: La mayoría de la materia prima lítica es de origen local aunque el análisis espectrométrico de elementos lantánidos, realizada en los EE.UU., sugiere que unas piezas de la cueva pueden corresponder a materia prima recogida a $25 \mathrm{~km}$ o más aguas arriba en el valle del Quípar y un raspador sobre radiolarita cuyo origen parece encontrarse a $40 \mathrm{~km}$ aguas abajo donde aflora dicha piedra. Para este último véase W. Zack, A. Andronikov, T. Rodríguez Estrella, M. López Martínez, M. Haber Uriarte, V. Holliday, D. Lauretta, M. J. Walker, (2013). De todas formas, hay muchos restos de la talla paleolítica que evidentemente tenían lugar en la cueva. datar?

C.I.C.G.: En la campaña pasada de 2012 aparecieron restos de una fogata. ¿Se ha podido

M.Walker: En 2012 y 2013 hemos excavado una zona profunda que demuestra la alteración 
térmica del sedimento y de huesos y sílex, y las investigaciones científicas en vías de realización en los EE.UU. y Canadá indican que la temperatura alcanzada por la fogata alcanzaba $550^{\circ} \mathrm{C}$. Prefiero no llamarla "hogar", palabra que implica bien una fosa o delimitación por piedras. El sedimento aporta indicios de alteración térmica según análisis micromorfológico microscópico, véase $\mathrm{D}$. Angelucci, D. Anesin, M. López Martínez, M. Haber Uriarte, T. Rodríguez Estrella, M. J. Walker (2013). La magnetoestratografía de todo el relleno de la Cueva Negra demuestra que este relleno tiene una fecha superior a 780000 años, porque el paleomagnetismo de los componentes del sedimento es de polaridad negativa, lo que indica que el sedimento se formaba durante el magnetocron de Matuyama, antes del cambio hace 780000 años a ser positiva o "normal" cuando comienza el actual magnetocron de Brunhes, véase G. R. Scott, L. Gibert, (2009). La luminiscencia por estimulación óptica de componentes del sedimento indica una antigüedad superior a los 500000 años (Dr. Jean-Paul Schwenninger de la Universidad de Oxford, comunicación personal). La bioestratigrafía y biocronología de los abundantes dientes excavados de roedores fósiles extintos denotan una antigüedad inferior a 990000 años pero desde luego superior a 700000 (concretamente, las especies Microtus (Allophaiomys/Euphaiomys) sp. cf. chalinei, Mimomyssavini, Pliomysepiscopalis, Microtus (Allophaiomys/Arvicola) sp. cf. deucalion, Microtus (Iberomys/Terricola/Pitymys) huescarensis huescarensis, Microtus (Iberomys) brecciensis brecciensis y Microtus (Stenocranius) gregaloides. La colección de dientes de roedores fósiles ha sido inspeccionada por el especialista Dr. Antonio Ruiz Bustos, profesor de la Universidad de Granada. La fauna mayor es coherente con el período según el estudio del paleóntologo Dr. Jan van der Made del Museo Nacional de Ciencias Naturales; el rinoceronte Stephanorhinus cf. etruscus, el équido Equusaltidens, el gamo Dama cf. nestiivallonnetensis, el ciervo gigante Megaloceros parecido al de la Cueva Victoria, precursores ambos probablemente de las especies del $M$. savini y $M$. verticornis del Pleistoceno Medio, el mamút Mammuthus (probablemente M. meridionalis), el macaco Macaca cf. sylvanus, el oso (Ursussp.), un hiénido (Hyaenidae), el bisonte (Bison cf. priscus) etc., véase M. J. Walker, M. V. López Martínez, J. S. Carrión García, T. Rodríguez Estrella, M. San Nicolás del Toro, J. L. Schwenninger, A. López Jiménez, J. Ortega Rodrigáñez, M. Haber Uriarte, J. L. Polo Camacho, J. García Torres, M. Campillo Boj, A. Avilés Fernández, W. Zack (2013). Estos datos cronológicos indican que entre 990000 y 780000 años la Cueva Negra tiene evidencia del fuego más antiguo de todos los yacimientos paleolíticos europeos. También se trata de la evidencia paleolítica más antigua en Europa para la presencia del hacha de mano achelense y la industria de útiles menores levaloisense-musteroide.

C.I.C.G.: La aparición de dicha fogata, ¿les da alguna pista sobre la utilización que hacía el Homo Heidelbergensis del sitio de Cueva Negra? Es decir, ¿era un lugar de taller, abrigo, o simplemente campamento estacional?

M.Walker: Evidentemente los resultados de la excavación demuestran que la cueva abrigaba todas estas actividades humanas de vez en cuando. Por otra parte, la acumulación del sedimento se producía por la invasión esporádica del agua durante crecidas del río, que alimentaba el pantano o lago delante de la boca de la cueva, habría hecho de la cueva un lugar incómodo debido al barrizal formado. También algunos datos tafonómicos implican la utilización del abrigo por animales y aves, sin duda durante ausencias humanas. Lo que la variedad de información analizada demuestra, eso sí, es el ingenio del Homo heidelbergensis hace casi un millón de años en el altiplano del Noroeste murciano, donde la cueva está a $740 \mathrm{~m}$ sobre el nivel del mar y abre hacia el Norte. Por lo tanto, podía aprovecharse de una amplia gama de recursos vitales en una zona entonces de notable biodiversidad, como de materias primas diversas que modificaba por técnicas variadas de la talla paleolítica. Todo esto demuestra la destreza manual, la aptitud tecnológica y la versatilidad cognitiva del Homo heidelbergensis hace casi un millón de años en España. 


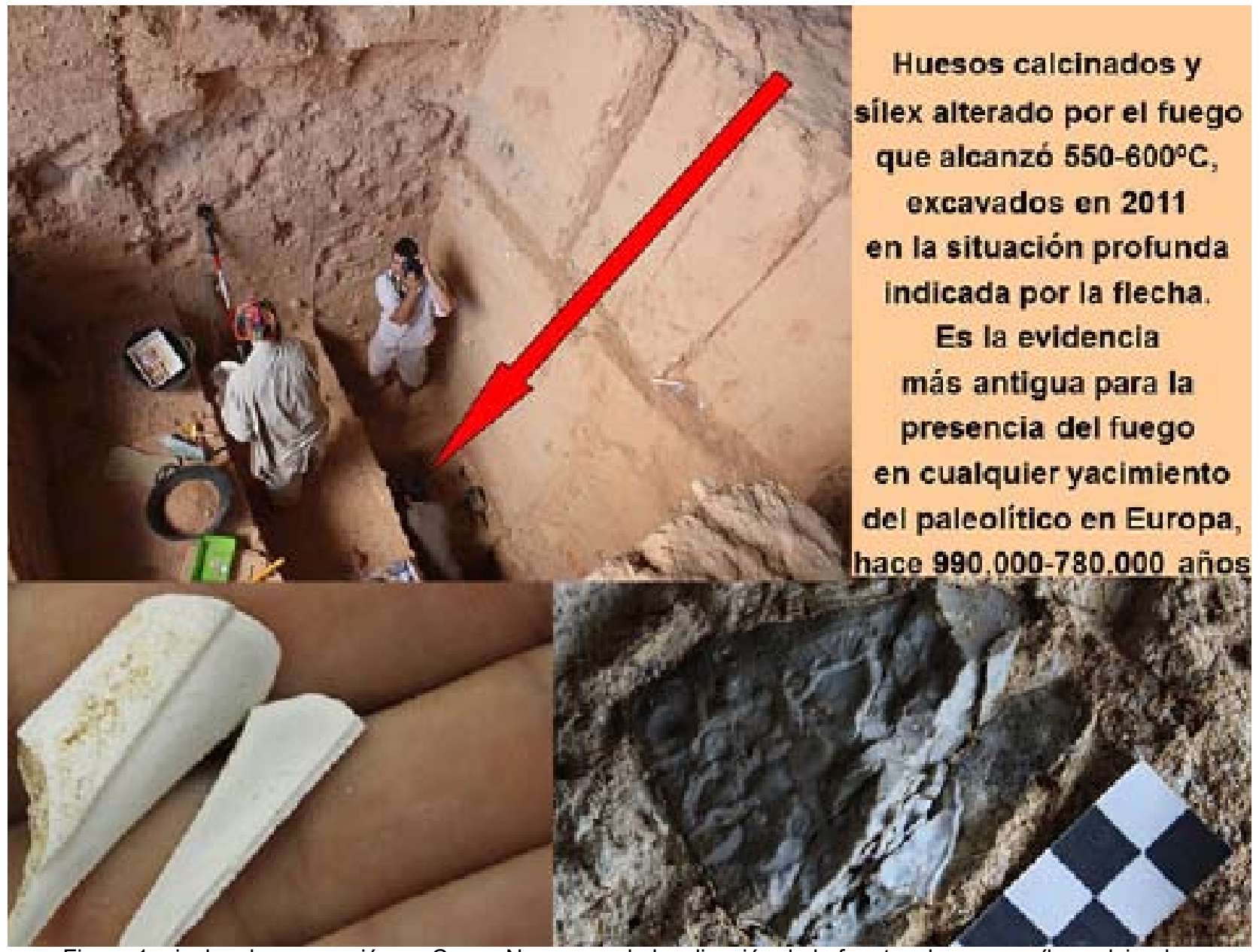

Figura 1: niveles de excavación en Cueva Negra, con la localización de la fogata y huesos y sílex calcinados.

Fuente: Michael Walker

C.I.C.G.: Pasando a Sima de Las Palomas, que es ya un yacimiento de renombre dentro de Historia de la Evolución Humana, puesto que se han descubierto varios esqueletos humanos, ¿nos podría explicar a cuántos individuos pertenecen estos restos y en qué contexto fueron encontrados estos restos?

M. Walker: Hemos excavado tres esqueletos articulados en estado de conexión anatómica, todos neandertales. Uno es de una mujer que hemos bautizado como Paloma", otro es de un niño (¿su hijo/a?) y el tercer esqueleto es de otro adulto. En "Paloma" y el niño la posición con manos juntas tocando la frente es la misma conocida en algunos otros esqueletos musterienses y neandertales. La flexión de los brazos se contrasta con la extensión de las piernas. Sin duda los cuerpos debieron estar en la citada posición antes del comienzo del rigor mortis. El conjunto se descubrió debajo de grandes piedras, acompañado por sílex musterienses, huesos quemados de fauna mayor (especialmente caballo) y patas de huesos articulados en conexión anatómica de patas de leopardo sin indicios del fuego. Es verosímil que los tres esqueletos fuesen tapados con piedras grandes para evitar la remoción por leopardos e hienas, y que las patas de leopardo se guardasen por los neandertales como trofeos o fruto de la caza. Huesos sueltos y dientes neandertales habían sido excavados en capas superiores y también en las escombreras abandonadas por los mineros que trabajaban en la cueva a principios del siglo XX o finales del XIX. El número mínimo de individuos neandertales representados por los restos es de nueve o diez, los tres esqueletos incluidos. Véase, M. J. Walker, M. V. López Martínez, J. Ortega Rodrigáñez, M. Haber Uriarte, A. López Jiménez, A. 
Avilés Fernández, J. L. Polo Camacho, M. Campillo-Boj, J. García-Torres, J. S. Carrión García, M. San Nicolás del Toro, T. Rodríguez Estrella, (2012). El riguroso análisis osteológico pone de relieve no sólo que se trata de un conjunto de restos del Homo neanderthalensis fuera de toda duda, sino que los individuos eran de talla inusualmente corta con respecto a los demás neandertales estudiados en Europa y Medio Oriente. Por otra parte, no han sido encontrados anteriormente restos neandertales tan completos en el litoral europeo del Mediterráneo. Caben diversas conjeturas sobre la estatura baja, por ejemplo la consanguineidad repetida, la deriva genética, o una adaptación biológica favorecida por la selección natural, véase, M. J. Walker, J. Ortega, K. Parmová, M. V. López, E. Trinkaus, (2011), M. J. Walker, J. Ortega Rodrigáñez, M. V. López Martínez, K. Parmová, E. Trinkaus, (2011), M. J. Walker, A. V. Lombardi, J. Zapata, E. Trinkaus, (2010), y M.J. Walker, J. Gibert, M. V. López, A. V. Lombardi, A. Pérez Pérez, J. Zapata, J. Ortega, T. Higham, A. Pike, J. L. Schwenninger, J. Zilhão, E. Trinkaus, (2008). La influencia de la dieta no puede ser descartada. Se ha detectado la presencia de caries en algunas piezas dentarias, véase M. J. Walker, J. Zapata, A. V. Lombardi, E. Trinkaus (2011). La identificación de fitolitos en el sarro de algunas piezas indica la ingestión de alimentos de origen vegetal, véase D. C. Salazar García, R. C. Power, A. Sanchis, V. Villaverde, M. J. Walker, A. G. Henry (2013). Esto pone en tela de juicio la conjetura popular de que los neandertales comían alimentos cárnicos principalmente.

\section{C.I.C.G.: ¿Pertenecen todos a la misma cronología?}

M. Walker: En 2013 se publicó la revisión cronológica que explica por qué se considera ahora que los restos esqueléticos neandertales son de hace aproximadamente 50000 años y no de hace 40.000 años, fecha más reciente como se pretendía en un artículo publicado en 2008. Véase M. J. Walker, et al (2012).

C.I.C.G.: Para marcarlos cronológicamente, ¿qué métodos de datación se han usado? Para ello, ¿ha sido necesario ponerse en contacto con otras universidades?

M. Walker: El artículo citado en la anterior pregunta, indica los métodos e investigadores de diversos centros que han colaborado en la aproximación científica rigurosa de la cronología. El método basado en el desequilibrio de isótopos del uranio ha aportado los resultados que más confianza inspiran, especialmente aquellos conseguidos por el Dr. Alistair Pike (actualmente profesor del Departamento de Arqueología de la Universidad de Southampton). El método sobre la base de la estimulación óptica de la luminiscencia aplicado por el Dr. Jean-Luc Schwenninger en la Universidad de Oxford ofrece un dato coherente. Por otra parte, las determinaciones del radiocarbono realizadas por el Dr. Tom Higham en Oxford no alcanzan la antigüedad verosímil del conjunto que está más allá del alcance de esta metodología. El asunto es tratado con detalle en M. J. Walker, et al (2012).

C.I.C.G.: ¿Cuál es su teoría sobre la deposición de estos cuerpos? ¿Cree que pertenecen a individuos enterrados concienzudamente, con una técnica y ritual, o simplemente una vez muertos fueron depositados en la Sima?

M. Walker: En relación con la información de la respuesta a la sexta pregunta, es verosímil conjeturar el enterramiento intencionado de "Paloma", el niño y el otro esqueleto articulado, quizás para evitar la remoción de los cadáveres por leopardos e hienas o para mantener la higiene (sofocando así el mal olor de la putrefacción), aunque no se puede descartar la posibilidad de un derrumbe de piedras mientras los tres dormían. La posición de las manos tocando la frente sugiere la disposición intencionada de los cuerpos antes del comienzo del rigor mortis y puesto que ha sido comentada en otros esqueletos excavados del musteriense o neandertales podría tratarse de una práctica social. Conjeturar "ritos" nos lleva al mundo de fantasía novelística incoherente con 
la ciencia arqueológica y paleoantropológica. La ciencia se limita a ensayar bien la refutación o la corroboración de hipótesis de trabajo relacionadas con modelos establecidos que explican las regularidades irregulares o las irregularidades regulares en la disposición espacio temporal o el comportamiento de observaciones materiales compatibles con otras nuevas. Cuánto más prosaica y sencilla la hipótesis a comprobar, tanto mejor.

C.I.C.G.: A parte de la aparición de "Paloma" y el resto de individuos, ¿qué más hallazgos se están encontrando en las campañas de excavación?

M. Walker: Como se ha mencionado en la sexta pregunta, hay restos fragmentarios de huesos o dientes que corresponden a un número mínimo de seis o siete individuos neandertales, además de los tres esqueletos articulados representados por "Paloma", el niño y el otro adulto. Hay un número considerable de restos paleolíticos musterienses y paleontológicos sobre todo de fauna mayor, especialmente caballo y artiodáctilos, además de leopardo y dientes de hiena e hipopótamo.

C.I.C.G.: ¿Se han encontrado tipologías iguales o parecidas de materiales entre Cueva Negra y Sima de las Palomas?

M. Walker: La respuesta corta es "No". Los útiles paleolíticos de la Sima de las Palomas son nítidamente musterienses, como es de esperar hace 50000 años. El musteriense no se identifica como tal hasta hace aproximadamente 300 000-250 000 años, en el Pleistoceno Medio tardío (por ejemplo, en el yacimiento holandés de Belvédère Maastrique). Por otra parte, los útiles menores de la Cueva Negra incluyen elementos parecidos a los de algunos yacimientos europeos del Pleistoceno Medio o Antiguo, que de vez en cuando han sido etiquetados como premusterienses, protomusterienses, protocharentienses, musterioides o musteroides, debido a la presencia esporádica de aspectos que "preconizan" el musteriense (por ejemplo, denticulados, raspadores o raederas de retoque marginal abrupto, puntas de Tayac, picos de pájaro o becs, babosas o limaces, o la extracción de lascas de manera centrípeta repetida levaloisense de núcleos discoideos pequeños). En su mayoría el conjunto de la Cueva Negra se parece más a otros del Pleistoceno Antiguo final (por ejemplo, Vallparadís en Cataluña) o Pleistoceno Medio antiguo (por ejemplo, Isernia La Pineta en Italia). Aunque en Cueva Negra, como en la mayoría de los yacimientos europeos del Pleistoceno Medio antiguo o Pleistoceno Antiguo final, la mayoría de las piezas modificadas sean de forma bastante irregular, la escasez de elementos grandes separa estos conjuntos del clásico oldowayense africano, caracterizado por grandes cantos tallados en pebbletools o choppingtools, grandes "picos", grandes discoideos, etcétera. Conviene mencionar que los primeros bifaces (hachas de mano y hendedores) tienen 1.7 millones de años de antigüedad en África donde ya en 1.3 los Dres Ignacio de la Torre y Rafael Mora han definido la técnica "levaloisense". Por consiguiente, no debería asustarnos la presencia del conjunto paleolítico achelense-levaloisense-musteroide de la Cueva Negra. Lo que demuestra la versatilidad cognitiva, la aptitud tecnológica y la destreza manual del Homo heidelbergensis en España hace casi un millón de años.

C.I.C.G.: ¿Considera que en algún momento los individuos de Cueva Negra pudieron tener relación con los moradores del Cabezo?

M. Walker: La respuesta corta es "No". Los individuos de Cueva Negra desparecieron antes de hace 780000 años. Los neandertales de la Sima de las Palomas vivieron hace tan sólo 50000. Así pues, si por "algún momento" se entiende el paso del tiempo estamos nosotros quince veces más cerca de la Sima de las Palomas que esta lo estuviera de la Cueva Negra. Por otra parte, las trayectorias evolutivas filogenéticas, tanto consideradas desde el enfoque craneológico como del ADN, quizás impliquen que ambos tuviesen más en común que tuvieran con nosotros. Según el análisis genético publicado en Nature en este mismo mes de enero del 2014, realizado por el equipo 
de investigación del célebre paleogenetista Dr. Svante Päabö que dirige el prestigioso Instituto "Max Planck" de Antropología Evolutiva de Leipzig, posiblemente hubiese separación en Homo heidelbergesis antes de hace un millón de años, con el resultado de un estirpe africano conducente al Homo sapiens (nosotros) y otro extra-africano conducente al Homo neanderthalensis (aunque la identificación esquelética inconfundible de ambos no está clara en el registro fósil hasta hace 200 000 años como mucho), véase K. Prüfer, F. Racimo, N. Patterson, Flora Jay, S. Sankararaman, S. Sawyer, A. Heinze, G. Renaud, P. H. Sudmant, C. de Filippo, H. Li, S. Mallick, M. Dannemann, Q. Fu, M.Kircher, M. Kuhlwilm, M. Lachmann, M. Meyer, M. Ongyerth, M. Siebauer, C. Theunert, A. Tandon, P. Moorjani, J. Pickrell, J. C. Mullikin,(2014).

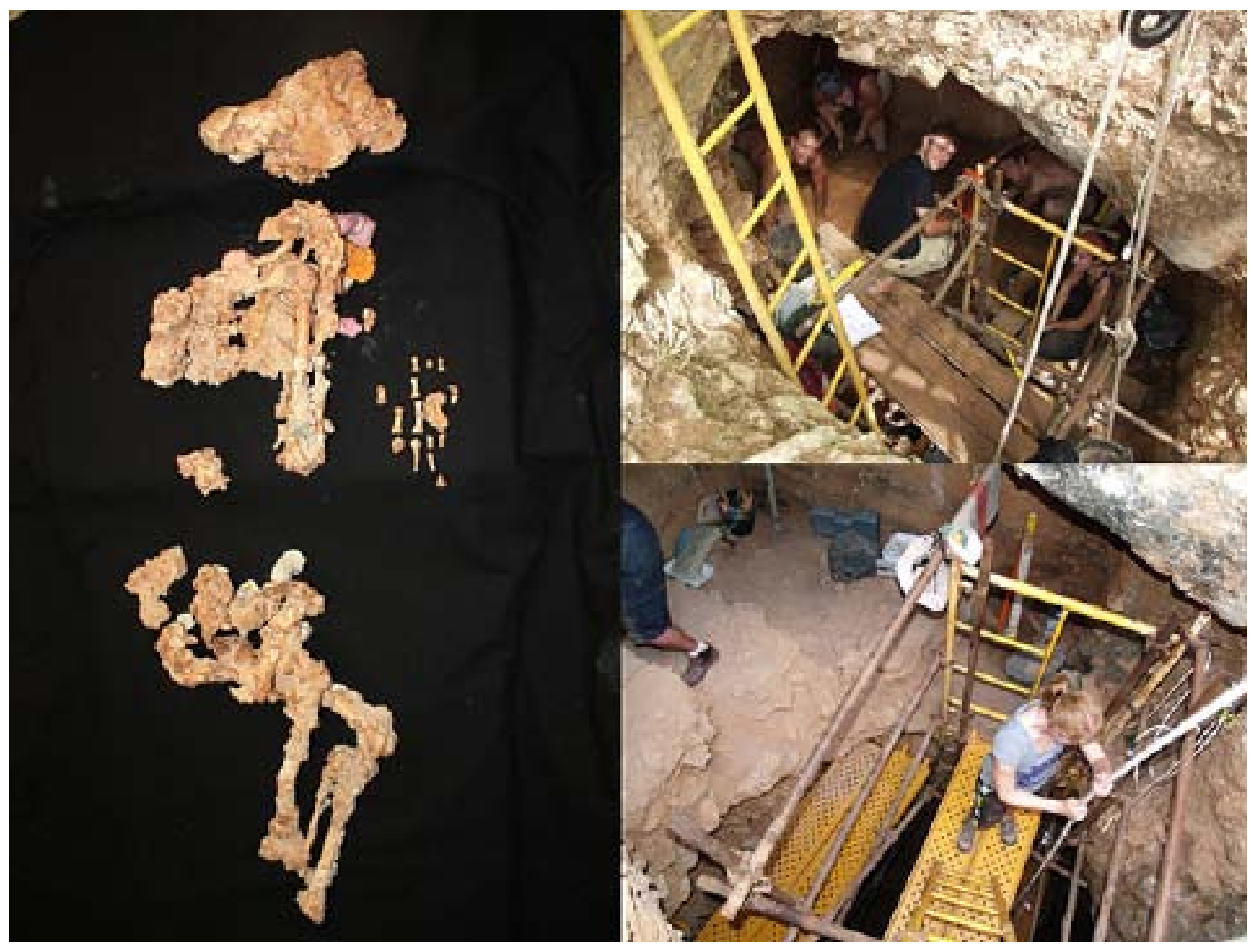

Figura 2: imagen del esqueleto articulado de Paloma y parte del equipo trabajando en la sima.

Fuente: Michael Walker

C.I.C.G.: ¿Cuál es el futuro que augura para las próximas campañas de excavación y estudio en ambos yacimientos?

M. Walker: Todavía queda mucho que excavar en los dos yacimientos. Son lugares especiales y sorprendentes, y cada campaña nos abruma con nuevas sorpresas. Auguro un porvenir brillante.

C.I.C.G.: Actualmente nos encontramos en una mala época económica que está afectando duramente al campo de la ciencia, ¿cuál es su opinión sobre el estado de la Arqueología y Antropología en la Región de Murcia? 
M. Walker: Aunque falten recursos económicos abundan el entusiasmo y la entrega de los profesionales, lo que es lógico teniendo en cuenta la enorme riqueza del registro del pasado en nuestra Región. Para aprovechar de ambos aspectos positivos opino que se debería diversificar la base socioeconómica de las actuaciones en Arqueología y Paleoantropología, promover organigramas de carácter mixto para fomentar la participación público-privada, y desmontar los rígidos sistemas actuales que tienden a reglamentar todo tipo de actuación desde la perspectiva de una mentalidad demasiado funcionarial acomodaticia, o incluso prepotente, que ve con reojo (cuando no con sospecha o desconfianza) y se cierra rápidamente ante cualquier sugerencia fuera de esquemas recibidos o establecidos sobre la oportunidad de desmantelar las barreras que impiden el flujo e intercambio, ágil y fluido, de propuestas innovadoras para desarrollar actuaciones de participación mixta, en situación de igualdad administrativa y organizadora, de entidades particulares y organismos o instituciones públicos.

C.I.C.G.: Muchas gracias por su tiempo y por su aportación a la investigación. Esperamos que su visión con respecto a las últimas dos preguntas se llegue a realizar.

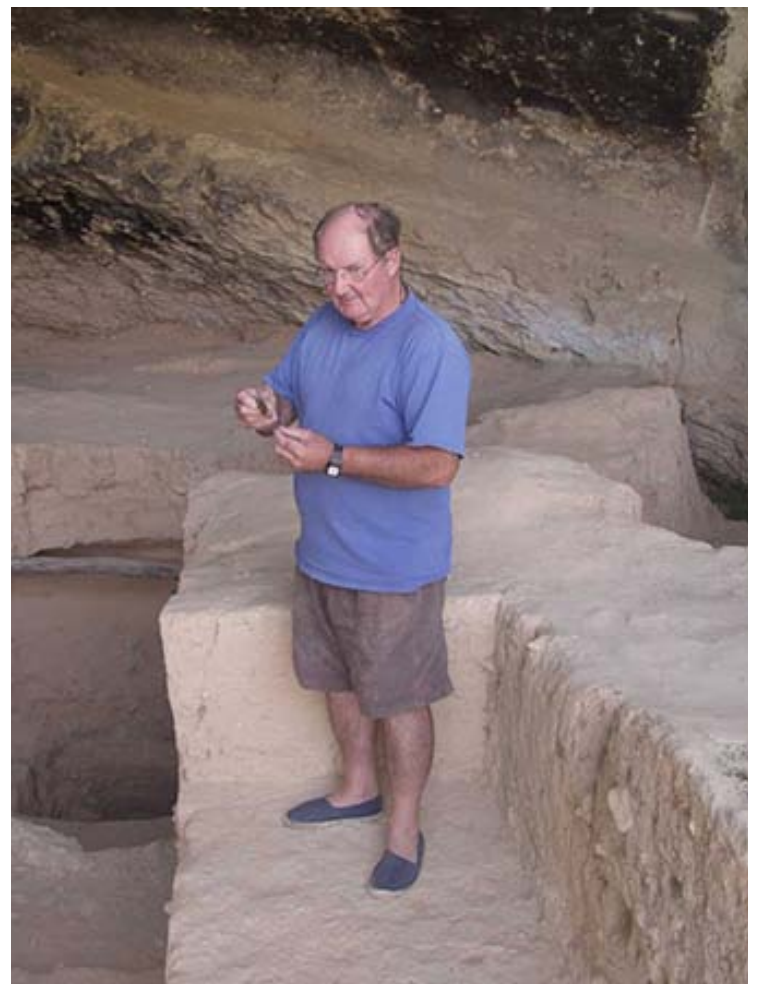

Figura 3: Michael Walker a pie de yacimiento en Cueva Negra.

\section{Bibliografía mencionada ${ }^{1}$}

Walker, M. J., Gibert, J., López, V. M., Lombardi, A. V., Pérez-Pérez, A., Zapata, J., Ortega, J., Higham, T., Pike, A., Schwenninger, J. L., Zilhao, J., Trinkaus, E., (2008). Late Neandertals in Southeastern Iberia: Sima de las Palomas del Cabezo Gordo, Murcia, Spain. Proceedings of the National Academy of Sciences USA 105, 20631-20636.

Scott G., Gibert, L., (2009). The oldest hand-axes in Europe. Nature 461, 82-85.

1 La bibliografía aparece por orden cronológico (no alfabético) por deseo expreso del entrevistado. Igualmente, se ha mantenido su sistema de citación. 
Walker, M. J., Lombardi, A. V., Zapata, J., Trinkaus, E., (2010). Neandertal mandibles from the Sima de las Palomas del Cabezo Gordo, Murcia, southeastern Spain. American Journal of Physical Anthropology 142, 261-272.

Walker M. J., Ortega, J., Parmová, K., López, V. M., Trinkaus, E., (2011). Morphology, body proportions, and postcranial hypertrophy of a female Neandertal from the Sima de las Palomas, southeastern Spain. Proceedings of the National Academy of Sciences USA 108, $10087-$ 10091.

Walker, M. J., Ortega, J., López, V. M., Parmová, K., Trinkaus, E., (2011). Neandertal postcranial remains from the Sima de las Palomas del Cabezo Gordo, Murcia, southeastern Spain. American Journal of Physical Anthropology 144, 505-515.

Walker, M. J., Zapata, J., Lombardi, A. V., Trinkaus, E., (2011). New evidence of dental pathology in 40,000 year old Neandertals. Journal of Dental Research 90, 428-432.

Walker, M. J., López, V. M., Ortega, J., Haber Uriarte, M., López Jiménez, Avilés Fernández, A., Polo Camacho, J. L., Campillo Boj, M., García Torres, J., Carrión García, J. S., San Nicolás del Toro, M., Rodríguez Estrella, T. (2012). The excavation of the burie darticulated Neanderthal skeletons at Sima de las Palomas (Murcia, SE Spain). Quaternary International 259, 7-21.

Walker, M. J., López, V. M., Carrión García, J. S., Rodríguez Estrella, T., San Nicolás del Toro, Schwenninger, J. L., López Jiménez, A., M., Ortega, J., Haber Uriarte, M., Polo Camacho, J. L., Avilés Fernández, A., Campillo Boj, M., García Torres, J., (2013). Cueva Negra del Estrecho del Río Quípar (Murcia, Spain): A late Early Pleistocene homininsite with an "Acheulo-LevalloisoMousteroid" Palaeolithicassemblage. Quaternary International 294, 135-159.

Zack, W., Andronikov, A., Rodríguez Estrella, T., López, V. M., Haber Uriarte, M., Holliday, V., Lauretta, D., Walker, M. J., (2013). Stone procurement and transport at the late Early Pleistocene site of Cueva Negra del Estrecho del Río Quípar (Murcia, SE Spain). Quartär, Internationales Jahrbuchzur Eiszeitalter- und Steinzeitforschung, International Yearbook for Ice Age and Stone Age Research vol. 60.

Angelucci, D., Anesin, D., López, V. M., Haber Uriarte, M., Rodríguez Estrella, T., Walker, M. J., (2013). Rethinking stratigraphy and site formation of the Pleistocene deposit at Cueva Negra del Estrecho del Río Quípar (Caravaca de la Cruz, Spain). Quaternary Science Reviews 80: 195-199.

Salazar García, D. C., Power, R. C., Sanchis, A., Villaverde, V., Walker, M. J., Henry, A. G., (2013). Neanderthal diets in central and southeastern Mediterranean Iberia. Quaternary International 318, 3-18.

Walker, M. J., López, V. M., Carrión García, J. S., Rodríguez Estrella, T., San Nicolás del Toro, Schwenninger, J. L., López Jiménez, A., M., Ortega, J., Haber Uriarte, M., Polo Camacho, J. L., Avilés Fernández, A., Campillo Boj, M., García Torres, J., Zack, W., (2013). Cueva Negra del Estrecho del Río Quípar (Murcia, Spain): A late Early Pleistocene hominin site with an "Acheulo-Levalloiso-Mousteroid". Palaeolithic assemblage. Quaternary International 294, 135159.

Prüfer, K., Racimo, F., Patterson, N., Jay, F., Sankararaman, S., Sawyer, S., Heinze, Gabriel Renaud, A., Sudmant, P., Filippo, C., LI, H., Mallick, S., Dannemann, M., FU, Q., Kircher, M., Kuhlwilm, M., Lachmann, M., Meyer, M., Ongyerth, M., Siebauer, M., Theunert, C., Tandon, A., Moorjani, P., Pickrell, J., Mullikin, J. C., (2014).The complete genome sequence of Neanderthal from the Altai Mountains. Nature 505, 43-49.

\section{Más bibliografía}

Algunas publicaciones recientes de divulgación científica en español sobre la Cueva negra y Sima de las Palomas: 
Walker, M. J., López, V. M., Haber Uriarte, M., Ortega, J., (2011). La Sima de las Palomas del Cabezo Gordo en Torre Pacheco: Excavación e Investigación en 2011. Verdolay. Revista del Museo Arqueológico de Murcia 13, 31-41. Número especial: Actas de los Encuentros sobre Arqueología y Paleontología, Museo Arqueológico de Murcia 21 a 25 de noviembre de 2011.

Walker, M. J., López, V. M., Haber Uriarte, M., Ortega, J., (2011). La Cueva Negra del Estrecho del Río Quípar en La Encarnación de Caravaca de la Cruz, Murcia, España: Excavación e Investigación en 2011. Verdolay. Revista del Museo Arqueológico de Murcia 13, 43-55. Número especial: Actas de los Encuentros sobre Arqueología y Paleontología, Museo Arqueológico de Murcia 21 a 25 de noviembre de 2011.

Walker, M. López Martínez, M. Haber Uriarte, A. López Jiménez, J. Ortega Rodrigáñez, A. Avilés Fernández, M. Campillo Boj (2011). "Dos yacimientos del Hombre fósil en Murcia: La Cueva Negra del Río Quípar en Caravaca de la Cruz y la Sima de las Palomas del Cabezo Gordo en Torre Pacheco. Segunda Parte. La Sima de las Palomas" Acta Científica y Tecnológica 19, 15-23.

Walker, M. J., López, V. M., Haber Uriarte, M., López Jiménez, A., Ortega, J., M., Avilés Fernández, A., Campillo Boj, M., (2011). Dos yacimientos del Hombre fósil en Murcia: La Cueva Negra del Río Quípar en Caravaca de la Cruz y la Sima de las Palomas del Cabezo Gordo en Torre Pacheco. Primera Parte. La Cueva Negra. Acta Científica y Tecnológica 18, 22-28.

Walker, M. J., López, V. M., Haber Uriarte, M., López Jiménez, A., Avilés Fernández, A., Campillo Boj, M., Ortega, J., (2012). Nuevos esqueletos neandertales y restos preneandertalenses de Murcia: La Sima de las Palomas del Cabezo Gordo (Torre Pacheco) y la Cueva Negra del Estrecho del Río Quípar (Caravaca de la Cruz). En D. Turbón, L. Fañanás, C. Rissech, A. Rosa (eds), Biodiversidad Humana y Evolución (Actas del XVII Congreso de la Sociedad Española de Antropología Física, Universidad de Barcelona, 2 a 4 de junio de 2011). Barcelona, Universitat de Barcelona and Sociedad Española de Antropología Física, 47-67. 



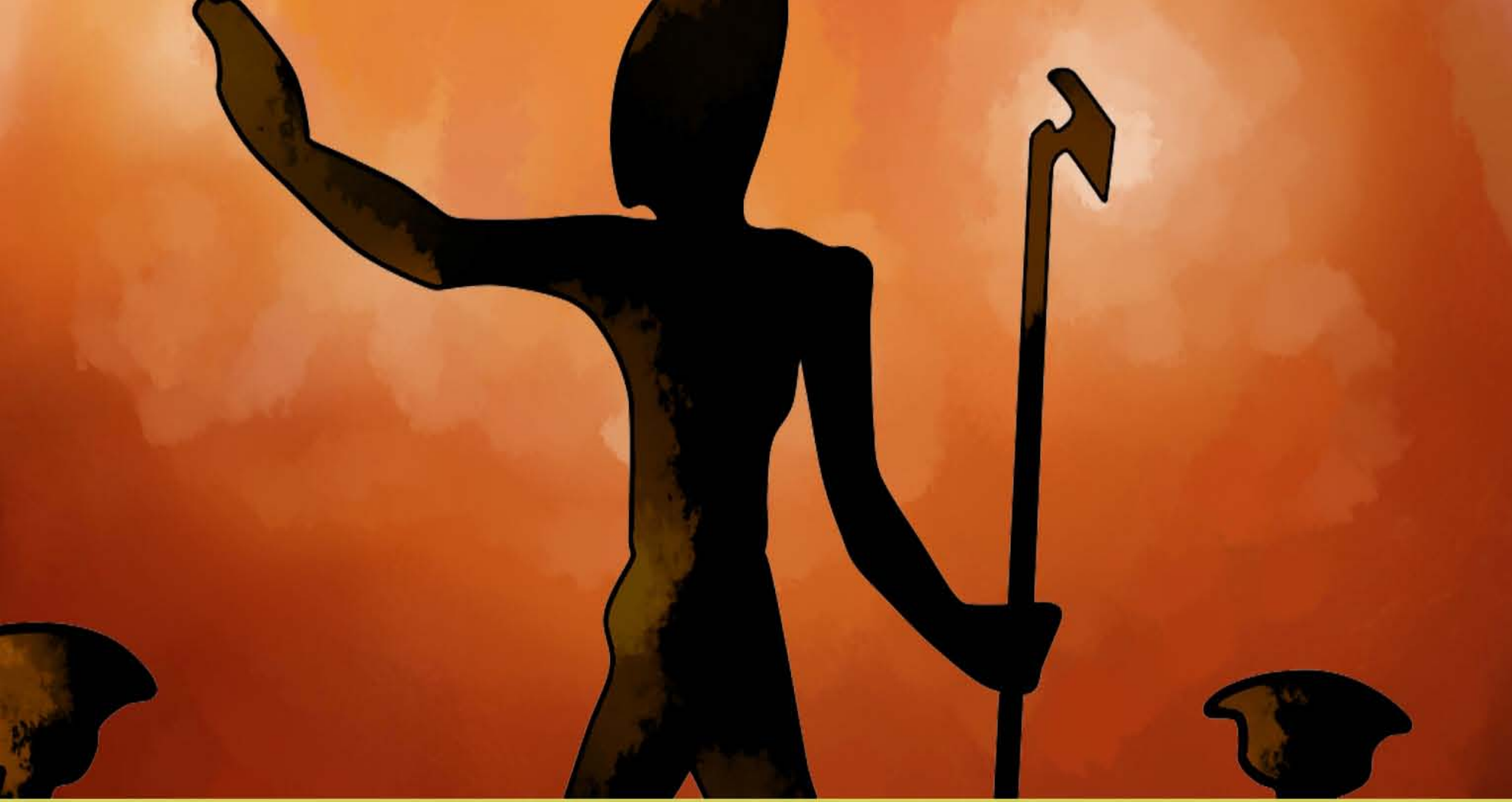

